\title{
Angioleiomyoma of the Tongue- A Rare Peculiar Entity
}

\section{Rawat G* and Aiyer HM}

Department of Pathology, Dharamshila Narayana Hospital, India

\section{Case Report}

Volume 4 Issue 1

Received Date: June 08, 2020

Published Date: July 01, 2020

DOI: $10.23880 /$ cprj-16000124 Department of Pathology, Dharam
Email: garima3103@gmail.com

\section{Abstract}

Angioleiomyomas (ALs) are benign mesenchymal tumors which were considered to be of smooth muscle origin, until the latest WHO classification (2016) of soft tissue tumors, in which it was reclassified as a tumor of perivascular origin. The majority of ALs occurs in the uterus, gastrointestinal tract and skin; AL of the oral cavity is infrequent, and AL of the tongue is particularly rare. These usually appear as solitary, slow-growing masses and are seldom observed in oral cavity. Here, we present a case of a fifty four year old male with a lesion on the left lateral border of the tongue, clinically mimicking a neurofibroma/ schwannoma. In this article we highlight the importance of scrupulous and detailed histopathological analysis and differential immunohistochemical studies which are essential for the diagnosis of AL from other spindle cell lesions.

Keywords: Oral cavity; Angioleiomyoma; Caldesmon; CD-34

Abbreviations: AL: Angioleiomyoma; WHO: World Health Organization; SMA: Smooth Muscle Actin.

\section{Introduction}

Angioleiomyoma (AL) is a benign neoplasm, considered a tumor of smooth-muscle origin until the most recent (2016) World Health Organization (WHO) classification of soft tissue tumors, when it was reclassified as a tumor of perivascular origin $[1,2]$. Earlier it was considered to be a vascular variant and a histological subtype of leiomyoma involving most often the lower extremities. ALs rarely involves the oral mucosa and they account for upto $65 \%$ of oral leiomyomas. Incidence in the oral cavity is as follows: labial mucosa followed by the tongue, buccal mucosa, hard and soft palate, and mandibular trigone $[3,4]$. The aetiology of AL is obscure especially in the oral cavity. However, various etiologies have been reported for origin of ALs including minor trauma, venous stasis and continuous mechanical trauma. Also, spontaneous development, genetic alterations and hormonal dysfucntions have been considered [2,5].

Genetic studies related to the origin of perivascular lesions are under evaluation also, mutations to the BRAF, NF1, NOTCH2 and NOTCH3 genes are under investigation [2]. Regarding hormonal changes as etiologic agent, the focus has been on estrogen and progesterone. Some studies focused on the role of progesterone in differentiation toward smooth muscle cells while in some estrogen and progesterone were shown to play different roles depending on the development of angioleiomyomas. More studies investigating the roles of ER and PgR in ALs are needed to reach some conclusion [4]. Few studies have also investigated the expression of sex hormone receptors in oral AL [2,6,7]. Here, we present a rare case of an angioleiomyoma involving the tongue.

\section{Case Report}

A fifty four year old male presented to the outpatient department with a history of swelling on the lateral border of the tongue gradually increasing in size. Intraoral examination revealed a well circumscribed bluish-red colored swelling on the left lateral border of the tongue. On palpation, the swelling was firm, painless, sessile, movable and approximately $2 \mathrm{~cm}$ in diameter. The remaining oral cavity was healthy. No cervical lymph nodes were detected during the extraoral 


\section{Clinical Pathology \& Research Journal}

examination. There was no significant medical history. The clinical differential diagnosis included Schwanoma/ Neurofibroma. The patient was planned for a core needle biopsy from the lesion for confirmatory diagnosis. We received a needle biopsy specimen composed of seven grey white soft tissue linear cores measuring 0.3 to $1 \mathrm{~cm}$ in length. Sections from the needle biopsy showed a tumor composed of bland spindle cells with an eosinophilic fibrillar cytoplasm (Figures 1A \& 1B). The tumour cells were in close proximity to proliferating blood vessels. Morphologic features were consistent with Low-Grade Spindle Cell Tumour-Possibly of smooth muscle origin (Figures 1C \& 1D). Subsequent immunohistochemical studies were performed and revealed the strong diffuse cytoplasmic staining for Smooth Muscle Actin (SMA) and Caldesmon (Figures 2A \& 2B). The tumour was immunonegative for Desmin, S-100, SOX-10, CD34 and CD31 (Figures 3(A-E). Thereafter, the patient underwent complete excision of the lesion.
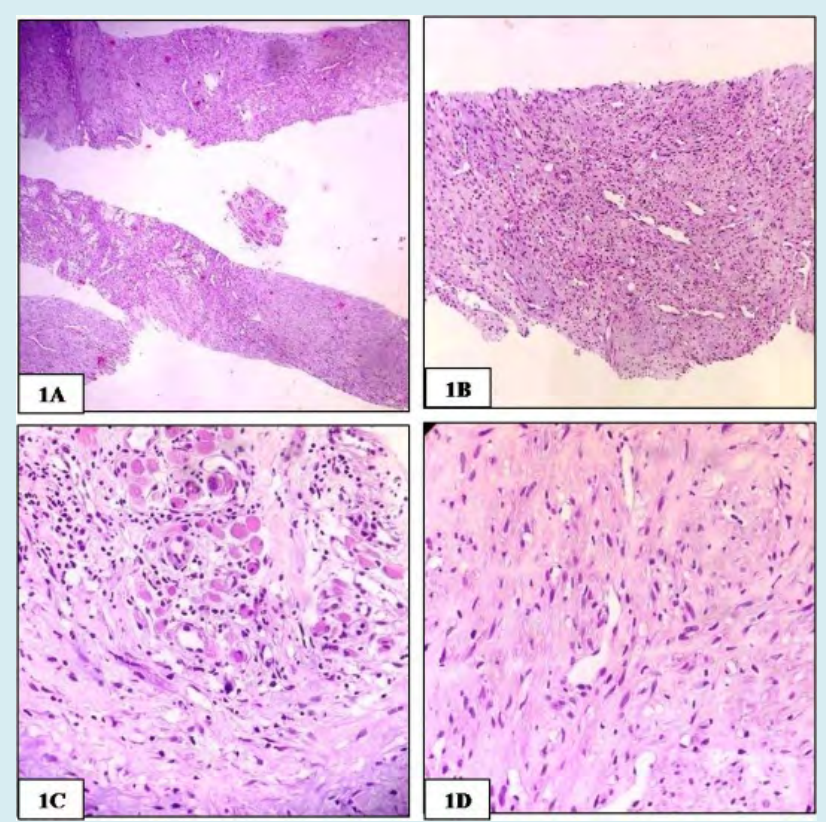

Figures 1(A-D): Tongue lesion images, A) Photomicrograph showing tumour composed of band spindle shaped cells. (HE-40x), B) Photomicrograph showing tumour cells in close proximity to proliferating blood vessels (HE-100x), C) Photomicrograph showing tumour cells with eosinophilic fibrillar cytoplasm and entrapped muscle. (HE-400x), D) Photomicrograph showing tumour cells in vicinity to blood vessels (HE-400x).
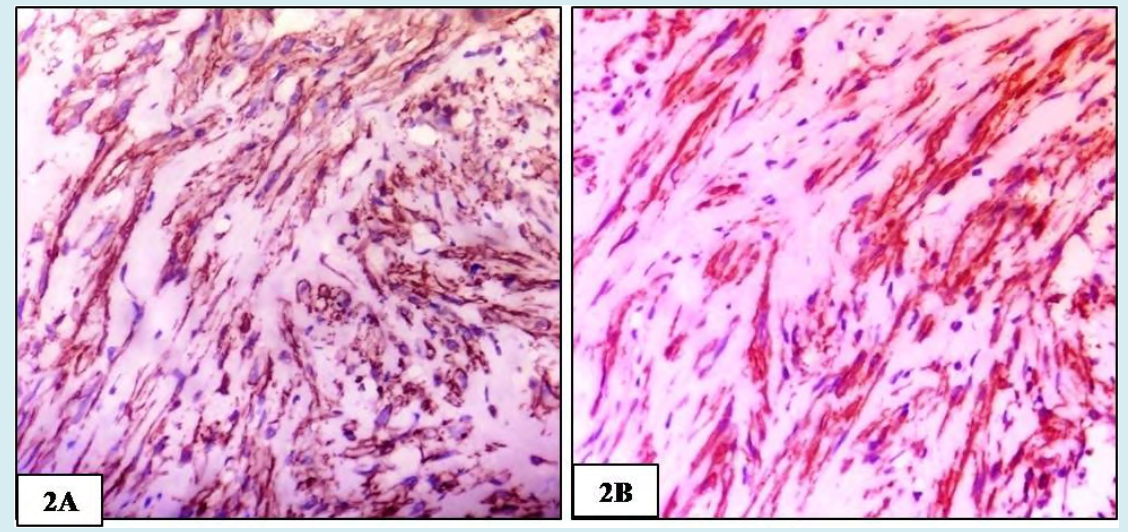

Figures 2(A\&B): Immunohistochemistry images, A) Photomicrograph showing tumour cells immunopositive for Caldesmon (400x) B) Photomicrograph showing tumour cells immunopositive for SMA (400x). 


\section{Clinical Pathology \& Research Journal}

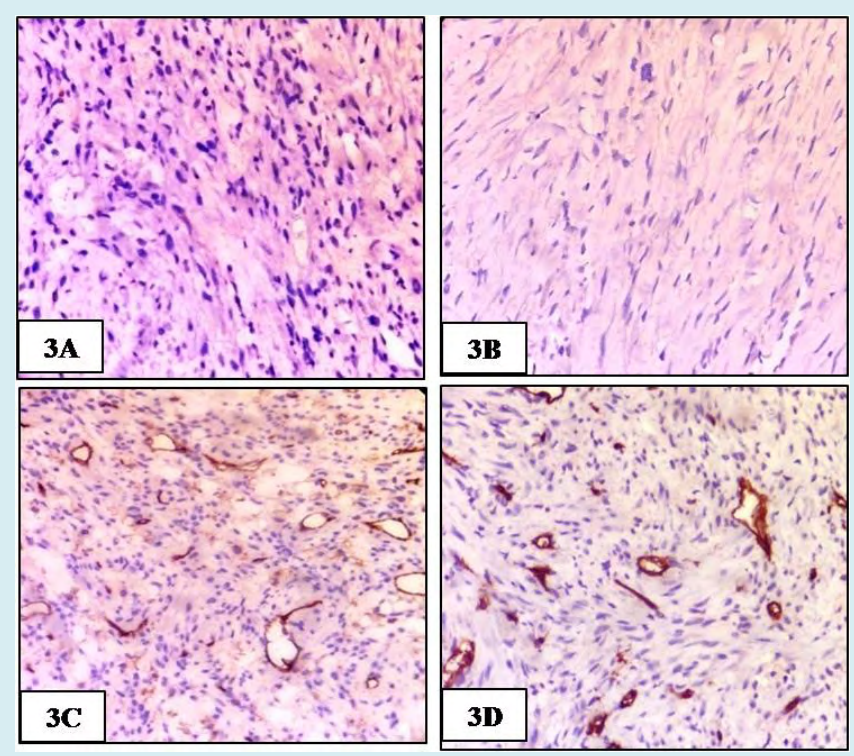

Figures 3(A-D): Immunohistochemistry images, A) Photomicrograph showing tumour cells immunonegative for S-100(400x) B Photomicrograph showing tumour cells immunonegative for SOX-10(400x)C) Photomicrograph showing tumour cells immunonegative for CD 34 (400x) D) Photomicrograph showing tumour cells immunonegative for CD 31 (400x).

\section{Discussion}

Angioleiomyoma of the oral cavity is an uncommon tumor. Hachisuga et al. reviewed 562 cases of AL and only 15 of these cases $(2.7 \%)$ occurred in the oral cavity. The oral lesions involved the following anatomic sites- lip, mandible, cheek and hard palate [8]. In another review by Brooks et al, the distribution of 121 oral ALs was surveyed [9]. The most frequent site of development was the lip (48.6\%), followed by the palate, buccal mucosa, tongue, mandible, buccal sulcus, labial sulcus, floor of the mouth and gingiva. Thus, these reports by various authors suggest that occurrence of $\mathrm{AL}$ of the tongue is rare $[6,8,9]$. The present case shows an unfamiliar lesion involving the tongue in the oral cavity. A review of literature on oral angioleiomyomas concluded that these tumours usually affect individuals from 4th to 6th decade of life. A slight male predilection is observed in oral angioleiomyomas $[2,10,11]$. The clinical findings in our case corroborate those described in previous literature with occurrence in an elderly male.

Most authors have observed that smaller tumors are asymptomatic whereas larger tumors produce mass effects such as difficulty in closing the mouth, chewing and swallowing. Tumors may occasionally get traumatized and ulcerated during oral function which may result in discomfort and pain. According to a review of the literature, a vascular appearance occurred in only $55.9 \%$ of angioleiomyomas, although they are vascular lesions. Malignant transformation has not been reported and the prognosis for ALs in the oral cavity is extremely good [4,6,10-12]. As most ALs have a non-specific clinical appearance and are painless, it is difficult to distinguish them from similar lesions, such as hemangioma, fibroma, angiofibroma, lipoma, and others $[6,9]$.thus, making the pre-operative diagnosis of head and neck angioleiomyomas challenging [13].

Histological examination is necessary for a definitive diagnosis. However, microscopic diagnosis may also be difficult at times, because the spindle cell proliferation, especially in core needle biopsy shares many similarities with neurofibroma, schwannoma, and fibroma. Immunohistochemical analysis represents an essential tool in reaching a definitive diagnosis of AL. The most common markers assessed to confirm the diagnosis of AL are SMA, HHF-35 actin, vimentin, CD34, desmin, and S-100. These immunohistochemical markers have assisted in the diagnosis in many cases as an adjunct to H\&E staining. The presence of SMA is especially useful for confirming smooth muscle origin of the tumor cells.

The monoclonal antibody to SMA reacts with the muscle cells of vessels and is useful in excluding a diagnosis of schwannoma, fibromatosis, fibrosarcoma, and malignant schwannoma since those tumor cells are negative for SMA. Another lesion which needs to be differentiated from ALs is myopericytoma, due to overlapping histopathological findings [2]. The characteristic immunohistochemical profiles of these two tumors when observed is immunopositivity for $\alpha$-SMA, HHF-35, and H-caldesmon, whereas desmin 


\section{Clinical Pathology \& Research Journal}

was negative in $75 \%$ myopericytomas and just $17.1 \%$ of angioleiomyomas. Therefore, desmin appears to be a useful marker for differentiation between these two lesions. Desmin may also be useful for differentiating angioleiomyomas from myofibromas in which the tumour cells are negative it.

S-100 marker is also useful for differentiating a diagnosis of AL from neoplasms of neural origin such as neurofibroma and neurilemmoma and also, granular cell myoblastomas (lesion with granular cytoplasm of its cells derived from Schwann cells), as the tumor cells of ALs fail to react to this protein, entirely divergent from what is observed in the above mentioned lesions $[2,5]$. The usual immunohistochemical profile of ALs is as follows: positive staining for SMA, Caldesmon, HHF-35 actin, vimentin, and desmin; and negative staining for S-100 protein and CD34 [6,11-14]. Similar results were obtained in our case. Therefore, each marker is a characteristic of a specific tissue, but its detection is useful only when applied in combination with clinical judgement and the expression of other markers.

It is crucial to differentiate AL from its malignant counterpart leiomyosarcoma, which is mainly characterized by undifferentiated mesenchymal cells or fibroblast- like and myofibroblast-like cells. Histologically differentiating AL from and low-grade leiomyosarcoma is arduous. Apart from the features of malignancy, immunohistochemical and molecular markers, such as proliferating cell nuclear antigen, B-cell lymphoma 2, cyclin-dependent kinase 4, p53, and mouse double minute 2 homolog, can also prove to be helpful $[3,14]$.

The treatment of angioleiomyoma is surgical excision. Though, angioleiomyomas are vascular, profuse bleeding is seldom observed. In most cases, this lesion can be easily removed by simple excision, similar to what was followed in the present case. The prognosis of surgical treatment of the lesion is typically excellent and recurrence has only been reported in cases of incomplete excision [3,7].

\section{Conclusion}

In conclusion, it is extremely rare for an angioleiomyoma to present in the head and neck. The diagnosis can only be made pre-operatively if a biopsy is undertaken. Angioleiomyoma is a benign tumor and surgery is almost always curative, and although it is rare in the head and neck, it is important to recognize that it can only be reliably diagnosed prior to surgery by biopsy and histopathologic examination. This article highlights the importance of histopathology and immunohistochemistry in the definitive diagnosis of Angioleiomyoma and distinguishing these tumours from other spindle cells lesions. Also, a review of previous literature concludes that angioleiomyomas of the tongue are a rare entity.

\section{References}

1. Fletcher CD (2014) The evolving classification of soft tissue tumours - an update based on the new 2013 WHO classification. Histopathology 64(1): 2-11.

2. Barbosa da SlA, Costa de MAM, O Teixeira de OP, Dantas da SEJ, da Costa MM (2017) Oral angioleiomyoma: case report and a review of current findings. J vasc bras 16(1): 68-72.

3. Matiakis A, Karakostas P, Pavlou AM, Anagnostou E, Poulopoulos A (2018) Angioleiomyoma of the oral cavity: a case report and brief review of the literature. J Korean Assoc Oral Maxillofac Surg 44(3): 136-139.

4. Inaba $T$, Adachi M, Yagisita $H$ (2015) A case of angioleiomyoma in the buccal space. Odontology 103(1): 109-111.

5. Menditti D, Laino L, Nastri L, Caruso U, Fiore P, et al. (2012) Oral angioleiomyoma: a rare pathological entity. In Vivo 26(1): 161-163.

6. Ishikawa S, Fuyama S, Kobayashi T, Taira Y, Sugano A, et al. (2016) Angioleiomyoma of the tongue: a case report and review of the literature. Odontology 104(1): 119122.

7. Yura S, Terahata S, Sugiguchi S (2011) A case of angiomyolipoma arising in the tongue. Case Rep Pathol 2011: 698139.

8. Hachisuga T, Hashimoto $\mathrm{H}$, Enjoji $\mathrm{M}$ (1984) Angioleiomyoma. A clinicopathologic reappraisal of 562 cases. Cancer 54(1): 126-130.

9. Brooks JK, Nikitakis NG, Goodman NJ, Levy BA (2002) Clinicopathologic characterization of oral angioleiomyomas. Oral Surg Oral Med Oral Pathol Oral Radiol Endodontol 94(2): 221-227.

10. Rawal SY, Rawal YB (2018) Angioleiomyoma (Vascular Leiomyoma) of the Oral Cavity. Head Neck Pathol 12(1): 123-126.

11. Tsuji T, Satoh K, Nakano H, Kogo M (2014) Clinical characteristics of angioleiomyoma of the hard palate: report of a case and an analysis of the reported cases. J Oral Maxillofac Surg 72(5): 920-926.

12. Barboza CAG, Ginani F, Leite-Segundo AV, Silva UH da (2013) Oral angioleiomyoma: a case report and considerations on differential diagnosis. J Bras Patol Med Lab 49(6): 429-432. 


\section{Clinical Pathology \& Research Journal}

13. Liu Y, Li B, Li L, Liu Y, Wang C, et al. (2014) Angioleiomyomas in the head and neck: A retrospective clinical and immunohistochemical analysis. Oncol Lett 8(1): 241-247.
14. Giudice A, Bennardo F, Buffone C, Brancaccio Y, Plutino FM, et al. (2019) Clinical and Immunohistochemical Features of Oral Angioleiomyoma: A Comprehensive Review of the Literature and Report of a Case in a Young Patient. Case Rep Dent 2019: 2498353. 